\title{
What do we know about the outcomes of tuberculosis contact investigations in NSW?
}

\section{Claudia C. Dobler}

Department of Respiratory and Environmental Epidemiology, Woolcock Institute of Medical Research, The University of Sydney

Email:cdobler@med.usyd.edu.au

\begin{abstract}
A recently conducted study on tuberculosis contact investigations in six Sydney tuberculosis clinics - that together managed $59 \%$ of all tuberculosis cases in NSW from January 2000 to December 2009 - found that the prevalence of tuberculosis among contacts was comparable to other low-incidence settings. However, only $9 \%$ of contacts with latent tuberculosis infection received treatment. This paper explores the results of the study, evaluating potential missed opportunities to prevent tuberculosis among contacts, and discussing the mechanisms in decision making about treatment of latent tuberculosis infection. In particular, the paper focuses on the challenges of tuberculin skin test interpretation among contacts who have received Bacille Calmette-Guérin vaccination and who were born in countries where tuberculosis is endemic.
\end{abstract}

Screening of contacts of people with active tuberculosis (TB) is a cornerstone of TB control in low-incidence countries such as Australia. ${ }^{1}$ Contact tracing aims to identify contacts with active TB or latent tuberculosis infection (LTBI) and to provide adequate treatment and follow-up. Active TB is evaluated by taking a history of clinical symptoms, performing a chest X-ray, and examining sputum samples if the chest X-ray is abnormal. A diagnosis of LTBI is based on a positive tuberculin skin test (TST), taking into account the pre-test probability of a contact having LTBI when interpreting the TST result. Contacts with a previously positive TST do not usually undergo a repeat TST, but the risk of having sustained a new infection from the current index case - depending on the infectiousness of the index case and duration and intensity of exposure of the contact - will be assessed by the reviewing physician. High-risk contacts will be offered LTBI treatment, and the remainder of the contacts will be followed-up with repeat chest X-rays for a minimum of 2 years.
In New South Wales (NSW), TB contact screening (including TSTs and preventive TB treatment) is guided by NSW Health policy directives. ${ }^{2-4}$ This paper will discuss the findings of a recently conducted study examining the risk of active TB among 14371 contacts of people with TB in NSW. ${ }^{5}$

Ethics apporval was obtained from the NSW Population and Health Services Research Ethics Committee (HREC/ 10/CIPHS/58).

\section{Active TB among contacts}

The study population of this retrospective cohort study comprised all persons who were screened as contacts of people with TB between January 2000 and December 2009 at TB clinics within the former Sydney West and Sydney South West Area Health Services. The six TB clinics within these two former Area Health Services together managed $59 \%$ of all TB cases in NSW during the study period. Cases of active TB among the study population were identified by linking a database containing details for all identified contacts (the Clinical Surveillance System) and the NSW Notifiable Diseases Database, which includes the TB registry and was used for the collection of notifiable disease case information in NSW until the implementation of the Notifiable Conditions Information Management System in 2010.

Of all contacts who were seen as part of TB contact investigations, $1.9 \%$ were diagnosed with active TB either at the time of the first screening or during follow-up. The prevalence of TB among contacts at the time of the initial screening (within 3 months after the first health-care contact) was $1.5 \%$ and thus consistent with the prevalence of active TB among contacts in other low-incidence settings. ${ }^{6}$ This relatively high yield for active TB at the time of initial screening ( $78 \%$ of contacts with active TB) compared to the lower yield during the subsequent follow-up period ( $22 \%$ of contacts with active TB were diagnosed during a mean follow-up period of 4.6 years) highlights the importance of ensuring at least one initial health-care screening visit for every contact at risk of having been infected.

\section{Latent TB infection: lost opportunities to prevent TB among contacts?}

In this study, $35 \%$ of all contacts with at least one TST performed had a TST $\geq 10 \mathrm{~mm}$ at the time of the initial screening visit or during follow-up and thus were identified 
as having LTBI. Applying the threshold for TST recommended in United States (US) guidelines ${ }^{7}(5 \mathrm{~mm})$ or

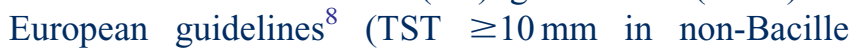
Calmette-Guérin (BCG)-vaccinated or BCG vaccinated $<12$ months of age; TST $\geq 15 \mathrm{~mm}$ in BCG-vaccinated $>12$ months of age) would have resulted in higher and lower estimated prevalence, respectively.

Only $9 \%$ of contacts with a positive TST in this study received treatment for LTBI. Do these findings imply that we are missing opportunities to prevent TB among contacts in NSW? Is comprehensive coverage of preventive treatment in contacts with evidence of LTBI what we should strive for? To answer these questions we need to take a closer look at those contacts that developed TB.

Of 273 contacts with active TB, 212 already had the disease at the beginning of contact screening and thus could not have been considered for treatment of LTBI. Of the remaining 61 contacts who developed TB during the follow-up period, details on TST results and/or chest X-rays were available for 45 . Of those, 30 (18 of whom were aged less than 35 years) had a TST $\geq 10 \mathrm{~mm}$ at the time of initial screening and did not receive LTBI treatment. Three of these 30 contacts were not given LTBI treatment because their chest X-ray was abnormal (including one contact aged less than 35 years), and active TB could not be excluded with certainty; they were followed up with a chest X-ray. Interestingly, 25 of the 27 contacts with a TST $\geq 10 \mathrm{~mm}$ and a normal chest X-ray who did not receive LTBI treatment were born overseas; the majority of these 25 contacts had received BCG vaccination.

Historically, US guidelines have recommended excluding from LTBI treatment persons aged 35 years and over with a positive TST but no other risk factors for TB. ${ }^{9}$ This recommendation followed a study that showed an association between age and increased risk of (potentially fatal) isoniazid-induced hepatitis. ${ }^{10}$ Subsequent US guidelines focus on screening and treatment of LTBI in high-risk populations only; the age cut-off no longer applies as this recommendation was directed at persons at low risk. ${ }^{1}$ The NSW policy directive on preventive therapy still applies an age cut-off of 35 years in considerations about who should be offered LTBI treatment. ${ }^{3}$

Given current NSW guidelines, at least the 17 contacts under the age of 35 years with a TST $\geq 10 \mathrm{~mm}$ and a normal chest X-ray at initial screening should have been considered for LTBI treatment. Sixteen of these contacts were born overseas and had received BCG vaccination. The findings highlight the challenge of TST interpretation in contacts who have received BCG vaccination and who were born in countries where TB is endemic; these contacts are likely to have been infected with TB previously. The decision to treat LTBI is a complex one in which the risk of progression to active TB (and associated morbidity and mortality) must be weighed against the risk of an adverse event from LTBI treatment. As the perceived potential risk of progression to active TB depends, amongst other things, on the likelihood of a false-positive TST (particularly in BCG-vaccinated contacts) and the likelihood of recent (compared to past) infection, this may explain a reluctance to offer treatment of LTBI to overseas-born contacts who have been BCG-vaccinated. Facing these uncertainties, it seems that the majority of chest clinic physicians in the study followed the principle 'primum nil nocere/first, do no harm' by avoiding the risk of adverse events from LTBI treatment.

\section{The role of interferon-gamma release assays in contact tracing}

TSTs have poor specificity in BCG-vaccinated individuals due to cross-reactivity with the antigens in the BCG vaccine. Interferon-gamma release assays (IGRAs), which have a much lower potential for false-positive results due to cross-reactivity, have been proposed as an alternative to the TST. However, IGRAs are currently not routinely used in NSW as an alternative or supplemental test in contact tracing. ${ }^{2}$ The reluctance to integrate IGRAs routinely as a diagnostic procedure in contact tracing is likely related to the fact that until recently there was little evidence that a positive IGRA result is a predictor of the risk of developing active TB. However, in recent years this association has been shown in several studies. Two meta-analyses published in 2012 examined the predictive value of IGRAs for progression to active $\mathrm{TB}$ : one concluded that the predictive value of IGRAs for development of TB is similar to the TST; ${ }^{11}$ the other found that IGRAs have a higher positive and negative predictive value for progression to active TB, especially in high-risk groups. ${ }^{12}$ In other countries with a low incidence of TB, such as the United Kingdom and the US, IGRAs have been introduced into guidelines on contact tracing. ${ }^{13,14}$ In these countries, IGRAs are used to verify a positive $\mathrm{TST}^{13}$ or as an alternative to the TST. ${ }^{14}$ The Australian National Position Statement on the role of IGRAs in the detection of LTBI, published in March 2012, also acknowledges the role of IGRAs as a supplementary test to confirm LTBI in low-risk individuals with a positive TST in the context of contact tracing. ${ }^{15}$ Due to the recently gained knowledge on the predictive value of IGRAs for active TB, which seems to be at least equivalent to the TST, and the higher specificity of IGRAs in BCG-vaccinated individuals, consideration should be given to integrating IGRAs, in addition to TSTs, into contact investigations in NSW.

\section{LTBI in overseas-born contacts}

The other challenge in TST interpretation (other than previous BCG vaccination) in contacts born in countries where $\mathrm{TB}$ is endemic is the uncertainty about the time of infection. LTBI treatment for recent infection is more 
beneficial than treatment for infection in the past, as the risk of progressing from LTBI to active TB disease is greatest in the 2 years following infection. ${ }^{16}$ If there is clear evidence of TB transmission from the index case, for example when an Australian-born child contact has a positive TST, this supports the assumption that recent infection could have occurred in all close contacts. However, if all household contacts of an index case were born in countries with a high TB incidence, it is difficult to establish whether recent transmission has occurred. It is likely that the reviewing physician takes uncertainty about the time of infection into account. This could explain the finding that overseas-born contacts with a positive TST often do not receive LTBI treatment. There is, however, evidence that treatment of LTBI in overseas-born close (household) contacts is cost-effective $e^{17,18}$ and worthwhile in terms of quality-adjusted life years. ${ }^{17}$ Indeed, further analysis of the NSW TB contact study shows that overseasborn contacts had a higher risk of developing active TB than Australian-born contacts (after adjustment for age, gender, TST size and whether preventive treatment was given) with odds ratios of 3.35 (95\% CI 1.84-6.10), 3.64 (95\% CI $2.48-5.35)$ and 5.79 (95\% CI 4.08-8.21) in overseas-born contacts from countries with a TB incidence of $<10,10-99$ and $\geq 100$ per 100000 population respectively (unpublished data). A more proactive approach to LTBI treatment in overseas-born high-risk contacts in NSW therefore seems indicated.

\section{Effectiveness of LTBI treatment}

In the NSW TB contact study, an estimated 3942 additional contacts with a TST $\geq 10 \mathrm{~mm}$ would have to have received LTBI treatment to prevent 38 cases of TB that occurred after initial screening among contacts with a TST $\geq 10 \mathrm{~mm}$; that is assuming $100 \%$ uptake, adherence, completion and effectiveness (unpublished data). However, in clinical practice, patient adherence to LTBI treatment is often a limiting factor in treatment effectiveness. Previous studies suggest low completion rates, ranging from 19\% (for isoniazid for 9 months) to $82 \%$ (for isoniazid for 6 months), with a completion rate of $75 \%$ for 6 months of isoniazid described at a major Sydney chest clinic. ${ }^{19}$ A Cochrane review of randomised controlled trials found an overall effect estimate of $60 \%$ for treatment of LTBI (taking into account patient adherence to treatment). ${ }^{20}$ While concerns about the overall effectiveness of LTBI treatment may be a reason for the reluctance of physicians to offer LTBI treatment, it is important to remember that randomised controlled trials have demonstrated that isoniazid has a $90 \%$ efficacy in preventing TB in infected contacts (and other individuals with LTBI) when taken properly. ${ }^{21}$

\section{Conclusion}

The prevalence of active TB among contacts in NSW is comparable to other settings with a low incidence of TB. Less than $10 \%$ of contacts with a TST $\geq 10 \mathrm{~mm}$ in the NSW
TB contact study received prophylactic treatment. Some missed opportunities to prevent TB have been identified, especially among young overseas-born high-risk contacts. Future challenges for TB contact tracing in NSW include achieving consistency in decision-making about LTBI treatment while taking individual patient factors into account, and defining the role of IGRAs in contact investigations.

\section{References}

1. American Thoracic Society. Targeted tuberculin testing and treatment of latent tuberculosis infection. Am J Respir Crit Care Med 2000; 161: S221-47.

2. NSW Health. Tuberculosis Contact Tracing. Policy Directive PD2008_017. Available at: http://www.health.nsw.gov.au/ policies/pd/2008/pdf/PD2008_017.pdf (Cited 30 December 2012).

3. NSW Health. Preventive Therapy. Policy Directive PD2005_072. Available at: http://www.health.nsw.gov.au/ policies/PD/2005/pdf/PD2005_072.pdf (Cited 30 December 2012).

4. NSW Health. Tuberculin Skin Testing. Policy Directive PD2009_005. Available at: http://www.health.nsw.gov.au/ policies/pd/2009/pdf/PD2009_005.pdf (Cited 30 December 2012).

5. Dobler CC, Marks GB. Risk of tuberculosis among contacts in a low incidence setting. Eur Respir J (in press).

6. Fox GJ, Barry SE, Britton WJ, Marks GB. Contact investigation for tuberculosis: a systematic review and meta-analysis. Eur Respir J 2013; 41(1): 140-56. doi:10.1183/09031936.00070812

7. American Thoracic Society/Centers for Disease Control and Prevention/Infectious Diseases Society of America. Controlling tuberculosis in the United States. Am J Respir Crit Care Med 2005; 172(9): 1169-227. doi:10.1164/rccm.2508001

8. Erkens CG, Kamphorst M, Abubakar I, Bothamley GH, Chemtob D, Haas W et al. Tuberculosis contact investigation in low prevalence countries: a European consensus. Eur Respir J 2010; 36(4): 925-49. doi:10.1183/09031936.00201609

9. American Thoracic Society, American Lung Association, Center for Disease Control. Preventive therapy of tuberculosis infection. Am Rev Respir Dis 1974; 110: 371-4.

10. Kopanoff DE, Snider DE, Jr, Caras GJ. Isoniazid-related hepatitis: a U.S. Public Health Service cooperative surveillance study. Am Rev Respir Dis 1978; 117(6): 991-1001.

11. Rangaka MX, Wilkinson KA, Glynn JR, Ling D, Menzies D, Mwansa-Kambafwile J et al. Predictive value of interferongamma release assays for incident active tuberculosis: a systematic review and meta-analysis. Lancet Infect Dis 2012; 12(1): 45-55. doi:10.1016/S1473-3099(11)70210-9

12. Diel R, Loddenkemper R, Nienhaus A. Predictive value of interferon-gamma release assays and tuberculin skin testing for predicting progression from latent TB infection to disease state: a meta-analysis. Chest 2012; 142(1): 63-75. doi:10.1378/ chest.11-3157

13. National Institute for Health and Clinical Excellence. NICE clinical guideline 117. Tuberculosis: clinical diagnosis and management of tuberculosis, and measures for its prevention and control. London; 2011. 
14. Mazurek GH, Jereb J, Vernon A, LoBue P, Goldberg S, Castro K. Updated guidelines for using Interferon Gamma Release Assays to detect Mycobacterium tuberculosis infection - United States, 2010. MMWR Recomm Rep 2010; 59(RR-5): 1-25.

15. National Tuberculosis Advisory Committee. Position statement on interferon-gamma release assays in the detection of latent tuberculosis infection. Commun Dis Intell 2012; 36(1): 125-31.

16. Ferebee SH. Controlled chemoprophylaxis trials in tuberculosis. A general review. Bibl Tuberc 1970; 26: 28-106.

17. Tan MC, Marra CA, Sadatsafavi M, Marra F, Morán-Mendoza O, Moadebi $\mathrm{S}$ et al. Cost-effectiveness of LTBI treatment for TB contacts in British Columbia. Value Health 2008; 11(5): 842-52. doi:10.1111/j.1524-4733.2008.00334.x
18. Dasgupta K, Schwartzman K, Marchand R, Tennenbaum TN, Brassard P, Menzies D. Comparison of cost-effectiveness of tuberculosis screening of close contacts and foreign-born populations. Am J Respir Crit Care Med 2000; 162(6): 2079-86.

19. Dobler CC, Marks GB. Completion of treatment for latent tuberculosis infection with monthly drug dispensation directly through the tuberculosis clinic. PLoS ONE 2012; 7(11): e48900. doi:10.1371/journal.pone.0048900

20. Smieja MJ, Marchetti CA, Cook DJ, Smaill FM. Isoniazid for preventing tuberculosis in non-HIV infected persons. Cochrane Database Syst Rev 2000; (2): CD001363.

21. Comstock GW. How much isoniazid is needed for prevention of tuberculosis among immunocompetent adults? Int J Tuberc Lung Dis 1999; 3(10): 847-50. 